\title{
UNA NUEVA ALTERNATIVA NUMÉRICA PARA LA SOLUCIÓN DE LA ECUACIÓN UNIDIMENSIONAL DE RICHARDS: ESTUDIO DE DRENAJE E INFILTRACIÓN DE FLUIDOS EN LA ZONA NO SATURADA
}

\author{
Evelyn Álvarez Sierra, Lilliam Álvarez Díaz y Maria Teresa Alonso González
}

\section{RESUMEN}

En el trabajo se comparan dos métodos numéricos para resolver el modelo unidimensional de infiltración y drenaje de agua en la zona no saturada en medios porosos, el cual es modelado respecto al contenido de humedad utilizando la ecuación no-lineal de Richards. El primer método está basado en el método clásico de Diferencias Finitas y el segundo en el método de Líneas combinado con el código DASSL para la solución de las ecuaciones diferencialesalgebraicas resultantes. Se muestra que el último método proporciona una vía numérica eficiente para la solución de problemas de EDPs que tienen un comportamiento singular de shock u ondas viajeras, como es el caso de la ecuación de Richard, los cuales se pueden resolver numéricamente con éxito sólo utilizando esquemas muy estables. Los métodos numéricos discutidos en el trabajo se aplican a dos tipos de suelos reales: Yolo Light Clay y Brindabella Silty Clay Loam, usando las propiedades hidráulicas referidas en Broadbridge y White [16]. Para validar el modelo, se comparan los perfiles de humedad con los resultados reportados por Warrick, Lomen e Islas [18]. Igualmente, se demuestra con datos reales la ventaja de resolver numéricamente el comportamiento del flujo unidimensional en la zona no saturada de un medio poroso. Finalmente, el modelo propuesto y los resultados numéricos obtenidos posibilitan brindar un pronóstico sobre la utilización de recursos hídricos para el caso de riego en agricultura y también para el transporte de contaminantes.

Palabras Clave: Ecuación De Richards, Drenaje, Infiltración De Fluidos, Zonas Saturadas. 